OPEN ACCESS

Edited by:

Marco Laurati,

University of Florence, Italy

Reviewed by:

Alan Ranjit Jacob,

Indian Institute of Technology

Hyderabad, India

Brijitta Joseph,

Sathyabama Institute of Science and

Technology, India

Igor Potemkin,

Lomonosov Moscow State University,

Russia

*Correspondence:

Laura Alvarez

laura.alvarez-frances@mat.ethz.ch

Specialty section:

This article was submitted to

Soft Matter Physics,

a section of the journal

Frontiers in Physics

Received: 06 August 2021

Accepted: 28 September 2021

Published: 20 October 2021

Citation:

Fernandez-Rodriguez MA and

Alvarez L (2021) Microgels and

Nanogels at Interfaces and Emulsions:

Identifying Opportunities From a

Bibliometric Analysis.

Front. Phys. 9:754684.

doi: 10.3389/fphy.2021.754684

\section{Microgels and Nanogels at Interfaces and Emulsions: Identifying Opportunities From a Bibliometric Analysis}

\author{
Miguel Angel Fernandez-Rodriguez ${ }^{1}$ and Laura Alvarez ${ }^{2 *}$ \\ ${ }^{1}$ Department of Applied Physics, University of Granada, Granada, Spain, ${ }^{2}$ Department of Materials, ETH Zurich, Zurich, \\ Switzerland
}

In this work, we used Bibliometrix and Biblioshiny to perform a bibliometric qualitative and quantitative analysis on the main topic "microgels and nanogels", and the sub-topic "microgels and nanogels at interfaces and emulsions". Word-counting of the titles of the publications enabled a descriptive analysis of thematic trends. A more complex conceptual analysis used the co-occurrence of words in titles, clustered into research themes with links to other themes. A thematic map allowed to characterize the centrality and density of the themes within the topic. A similar clustering of co-authorship enabled the mapping of the collaborations. We identified in this way research opportunities theme- and collaboration-wise, such as the opportunity to bring more fundamental studies into biomedical applications and into Pickering emulsions stabilized with microgels.

Keywords: microgels, nanogels, interfaces, emulsions, bibliometric analysis

\section{INTRODUCTION}

One of the main responsibilities of a researcher at all academic stages is to have a comprehensive and critical overview of the state-of-the-art of their research field and/or topic, mostly acquired throughout bibliographic material. Nevertheless, the search of bibliographic material can be overwhelming when collecting and processing publications due to the ever-increasing publishing rates. Currently, the gathering of up-to-date bibliography is performed by using tools as Web of Science (Clarivate Analytics P.L.C.), Scopus (Elsevier), PubMed (National Institutes of Health, United States), or Google Scholar (Google), just to name a few. All of them allow searching for original manuscripts on a particular scientific topic through keywords.

The aforementioned web-based tools are often used to collect and store bibliographic entries into a personal database, yet, a comprehensive reading of each bibliographic entry is needed to acquire a true overview of the state-of-the-art of the corresponding field of study. Using a bibliometric engine helps in this endeavour and presents great potential to provide a wider overview of the published scientific bibliography on a certain topic. This overview is possible because the bibliometric engines allow batch processing of a particular bibliographic database, granting the researcher a versatile analysis and display tools of the bibliographic material. Thus, an up-to-date bibliographic database efficiently processed by a bibliometric engine might help to provide qualitative and quantitative analysis about the time evolution of topics, scientific interactions, and possible open challenges. Moreover, this kind of analysis of a specific topic might be helpful to foster collaborations among a variety of different research areas: from pure fundamental to applied approaches, but also across different fields. 
In this manuscript, we explore the possibilities of using Bibliometrix [1] as a bibliographic engine. Bibliometrix is an open-source tool written in $\mathrm{R}$, a statistical analysis-oriented programming language. In particular, we use Biblioshiny, an easy-to-use website front-end for Bibliometrix. We have chosen as a case of study the main topic of microgels and nanogels, two nomenclatures referring to the same broad system of gels in the form of micro- and nanoparticles. By searching the topic "microgel* OR nanogel*" in Web of Science with no other constraints, the website collects and shows all bibliography items published since 1900, with the words microgel or nanogel and their deriving words appearing in either its title, abstract, or keywords. Within that search and constraining up to the year 2020, Web of Science provides 11,750 items, and therefore reading or sorting all of them becomes a difficult task. Thus, we propose the use of Biblioshiny [1] to analyze and extract useful information in an automated way.

The goal of the article is three-fold: 1) provide an overview of the versatility of Biblioshiny, by processing and displaying the information gathered by this bibliometric engine 2) evaluate the overall impact within the main topic of "microgels and nanogels" of the sub-topic "microgels and nanogels at interfaces and emulsions" 3) visualize the main research themes of the topic and current collaboration networks for the past 10 years, identifying current challenges and opportunities, both themeand collaboration-wise. We used Web of Science to collect the bibliographic dataset, but the methodology applies to databases generated by other scientific bibliography search engines. Furthermore, this bibliometric tool provides useful data on the scientific performance of authors and journals, including a variety of rankings based on the h-index constrained to the publications being analyzed, which we encourage to be explored. Nevertheless, here we focus on an analysis of research themes and current collaboration networks. We think that this approach can provide significantly faster overviews of a main topic of research and subtopics, in combination with the usual reviews. In this specific case, there are 596 reviews within the main topic published between 2010 and 2020, and 196 reviews between 2019 and 2020. Therefore, we encourage trying the approach laid in this manuscript.

\section{METHODOLOGY}

The methodology comprises two main steps: 1) gathering a raw database from Web of Science, and 2) processing it with Biblioshiny. Various treatments and filtering of the data are necessary at each step to ensure an efficient and useful database analysis.

\subsection{Database Generation and Treatment}

We performed two main searches in the Topic field of Web of Science constraining to publications up to the year 2020: 1) a general search using the terms "microgel* OR nanogel*", with 11,750 results in total, and 2) a search using the terms " [microgel ${ }^{\star}$ AND (interface ${ }^{\star}$ OR emulsion ${ }^{\star}$ )] OR [nanogel ${ }^{*}$ AND (interface* OR emulsion ${ }^{\star}$ )]", with 1908 results in total.
This second search will serve as an example of gathering information of a sub-topic in a broader topic, aiming for interfacial science aspects within the microgel and nanogel topic. The use of asterisk ensures the search of the main words and its deriving terms.

We then export full records of our bibliographic items from Web of science in plain text, containing the most information possible for the bibliometric analysis. Currently, Web of Science only allows exporting plain text files with up to 1,000 bibliography items at once. Therefore, we exported several files by selecting 1,000 items using the export menu in Web of Science. Ultimately, we end up with several plain text files containing the entire record of each bibliography item of the search in Web of Science.

Next, we pre-process the files as Biblioshiny does not distinguish between plurals and other derivations of words in many of its tools. Therefore, we identify significant words for our analysis and process the plain text files to replace singular forms with plural forms. Here we used the shell command "sed" to replace the command line by batch processing many plain text files at once. In this way, we find all words deriving from $\mathrm{gel}$, interface, emulsion and particle and replace singular forms by plural forms. Once this is performed, all the text files can be compressed into a zip folder which will be imported to Biblioshiny. We refer here to the Biblioshiny website (www. bibliometrix.org/Biblioshiny.html) for other tutorials and instructions on how to use this tool regarding the preformatting and importing databases to Biblioshiny.

\subsection{Bilioshiny Workflow}

Dataset filtering: once the database file is imported to Biblioshiny, the next step is to filter the document type from the following categories: articles, books, book chapters, reviews, data papers, proceedings, retractions, corrections, editorials, letters, meeting abstracts, news items, notes, early access papers, and reviews. In this study, we only excluded the early access papers as we were also limited to items published until 2020. It is also possible to filter by the number of citations.

Dataset descriptive analysis: the first descriptive analysis that we extracted is the annual production, a feature already available in many scientific web search engines. Next, we performed another descriptive analysis based on word counting and word evolution in the titles of the bibliography items. We obtain a word cloud with the most common 50 words defined by the word's frequency, and the temporal evolution of the most common 15 words based on occurrences per year. This word-based analysis is available for titles, abstracts, and keywords. Furthermore, all results are available both as plots and tables that can be exported for further processing.

Dataset conceptual structure: we performed a scientific mapping analysis of themes, also known as conceptual structure, based on the co-occurrence of words in the titles of the bibliography items. First, it obtains the number of cooccurrences between two words across titles. Next, the results are normalized by "association", also known as proximity index, which allows comparing similarities between co-occurrences. Then, the terms are clustered with a Louvain algorithm [3]. Finally, we plot the 20 more relevant words and their links by 


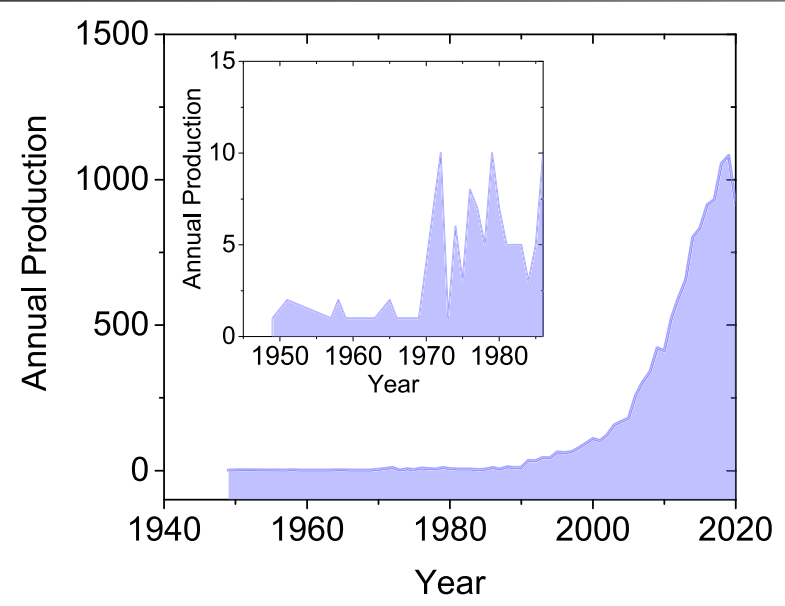

FIGURE 1 | Bibliographic annual production. Annual number of articles produced in the topic of microgels and nanogels, from the first paper in 1949 defining the term microgel until 2020. The inset represents the annual production from 1949 to 1986 .

a Fruchterman \& Reingold force plot [4]. Furthermore, we produced a thematic map also based on co-occurrence of words [5]. This time the terms are first stemmed by the Porter's algorithm to account for derivation of words in English [6], and the terms are clustered by the K-means algorithm [7] to find clusters that represent possible research themes. 1,000 words are considered with a minimum cluster frequency of five per thousand of bibliography items. These research themes are plotted in $2 \mathrm{D}$ as dots with the corresponding five more relevant words, where the $x$ axis is the centrality, measuring how the terms of a theme are shared (i.e., used) within other themes, while the $y$ axis is the density, measuring how strong are the co-occurrences of words within a theme [5]. We restricted to uni-grams, i.e., analysing appearances of single words.

Dataset social structure: we used the collaboration network analysis within the social structure category available in Biblioshiny. This tool performs a similar analysis like the one described above for co-occurrence of words, but this time for pairs of co-authors. In this way, identified clusters of collaborators are also reflected by the same color with thicker links corresponding to stronger collaborations. For this analysis, we included "isolated" authors whose co-authors do not appear in the plot because of the restriction in the total number of authors shown, i.e., 50.

\section{RESULTS AND DISCUSSION}

Biblioshiny, the front-end of Bibliometrix [1], can provide a quantitative analysis of the evolution of a research topic over time, but also a qualitative visualization of the trending themes around the chosen topic. We performed this analysis as a general historical evolution using the topic search "microgel* OR nanogel $^{*}$ in Web of Science, from the years 1949-2020. In
1949, Baker defined for the first time the term microgel as a particle that can be swollen in a good solvent [2]. As can be seen in the inset of Figure 1, where we represented the annual production of the main topic, the number of papers published after Baker defined the term remained rather low until the 80s. In 1986, Pelton and Chibante reported for the first time a very detailed synthesis route of microgels made of poly (N-isopropylacrylamide) hydrogel (pNIPAM) [8], and since then, many other monomers have been successfully used to synthesize the corresponding microgels [9].

At the time of writing the present work, the manuscript by Pelton and Chibante accumulates 943 citations according to Web of Science, 995 to Scopus, and 1,269 to Google Scholar. These high figures evidence its scientific impact, granting it the consideration of foundation of this research topic. Yet, it took around four more years for the research topic to start to increase significantly in bibliographic production, as seen in Figure 1. One of the most commonly studied features of pNIPAM microgels, shared by many other hydrogel-based micro and nanoparticles, is their responsiveness to stimuli, as temperature or $\mathrm{pH}$, which enables to collapse and swell the microgels in the dispersion solvent in a reversible way. This feature was discussed by Pelton in 2000 [10], accumulating 1,530 citations according to Web of Science, probably being one of the inflection points in the research topic, gathering the interest of the scientific community as reflected by the kick-off of scientific production that can be seen in Figure 1. It was around this time, in 1998, when Graham and Cameron distinguished between microgels and nanogels by their molecular weight "in the low thousands (nanogels) to many millions (microgels)" [11]. Nevertheless, we nowadays see that these terms usually refer to ranges of sizes that often overlap, and it seems a choice of nomenclature, as we will discuss later in the conceptual structure analysis.

The significant increase in the annual production after the discussion by Pelton in 2000 on the responsiveness to temperature and $\mathrm{pH}$ of microgels made of pNIPAM [10] suggests a natural response of the scientific community to these new findings. The $\mathrm{pH}$ - and thermo-responsiveness of pNIPAM microgels opened up new research directions to cargo-release and other biomedical applications. These first discoveries resulted in a clear increase of investigations focused on the development of microgels and nanogels using more bio-compatible smart materials. This new expansion of the field with the research for applications reflects in the timeevolution of the word-counting analysis of the titles of the publications (Figure 2). In Figures 2A,B we represent the word-counting analysis of the annual occurrences from 2010 to 2020, and a word cloud, both representing the main topic "microgels and nanogels", and in Figures 2C,D the corresponding results for the sub-topic "microgels and nanogels at interfaces and emulsions".

The restriction of the word-counting analysis to titles was motivated by the assumption that authors place there the words they consider the most interesting to appeal to the reader. Other possible choices for analysis are keywords (which tend to be more conservative to fit in a given research field), abstracts, and KeyWords Plus. The latter category belongs to Clarivate 

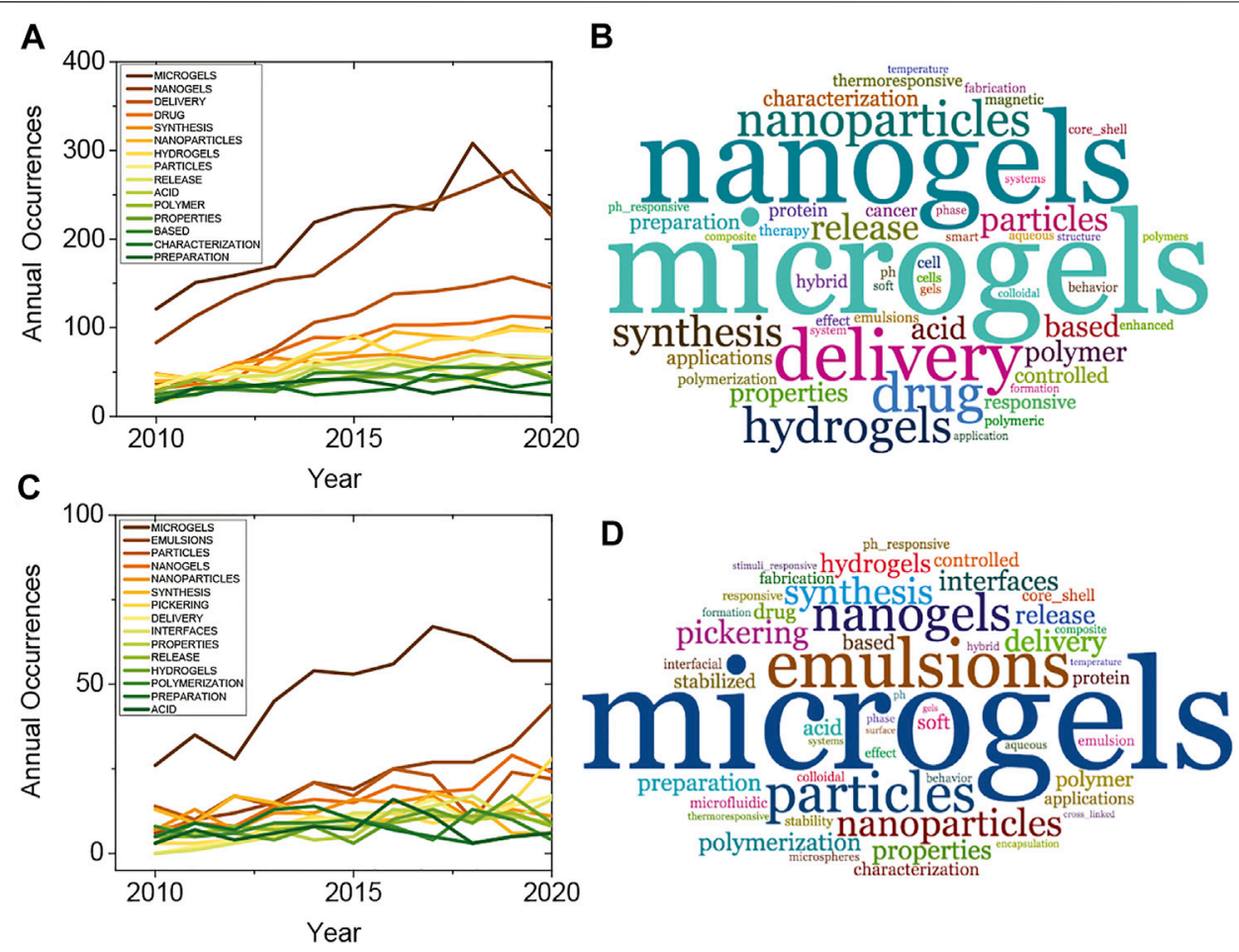

FIGURE 2 | Bibliographic word-counting analysis between the years 2010-2020. Annual occurrences of words and word-cloud for the topic "microgels and nanogels" (top) and "microgels and nanogels at interfaces and emulsions" (bottom) using (A, C) word dynamics representing the annual count for the 15 most repeated words. (B, D) word-cloud analysis representing the 50 most repeated words in the titles of the bibliography items.

Analytics, which according to Web of Science, KeyWords Plus represents "words or phrases that frequently appear in the titles of an article's references, but do not appear in the title of the article itself'. Articles before 1991 don't have them. In both main topic and subtopic searches, the dominant term repeated over the years is microgels (Figures 2A-C), with a variety of other terms related either to applications, properties and/or composition.

While the term microgels is followed closely by the term nanogels, especially in recent years, this term appears to be much less present in the sub-topic of "microgels and nanogels at interfaces and emulsions". In fact, the general overview of the "microgels and nanogels" field (Figure 2A) shows a tendency towards biomedical-related terms, such as delivery and drug.

Moreover, the similar increasing trend over time for terms such as synthesis, nanoparticles, and nanogels hints the interest in the optimization of the various fabrication protocols of the mentioned soft particles. In addition, the scientific production of the term microgel has decreased in the past couple of years, reaching the same occurrences as the term nanogel. Since the total number of publications increased (see Figure 1), this decrease might hint a change in the preferred nomenclature, or enough development of the field to not explicitly use the term microgel in the titles of the publications. In Figure 2C we show the word dynamics for the sub-topic "microgels and nanogels at interfaces and emulsions". Even though this specific sub-search might provide a bias towards the terms interfaces and emulsions, only the latter is present in a second place, followed by the terms nanogels, Pickering, and interfaces. Moreover, there is a clear increase within the last 5 years of the occurrences of the terms emulsions and Pickering, showing a bigger interest of using these particles in this specific type of emulsions. Moreover, the lower occurrence of the term nanogels hints to a choice of the term microgels within this sub-topic. The term delivery considerably drops with respect to the main topic, and drug is not present within this list, indicating the opportunity to further develop the biomedical applications by incorporating fundamental aspects of microgels and nanogels at interfaces and emulsions, important in drug delivery. Finally, for both cases there seems to be a rather continuous annual occurrence trend for terms related to characterization and composition, such as acid, base or polymer, transverse to all studies.

If we have a wider look at the 50 most important terms arising around each case (Figures 2B-D, main topic and interfaces/ emulsions sub-topic, respectively), we find differences that are biased by the topic search. However, ultimately, many other concepts are repeated that are intrinsically related to the microgel and nanogel properties and applications.

In order to expand the analysis and achieve insights on the relation between terms and their clustering into possible subtopics, we performed a conceptual structure analysis by analyzing the co-occurrence of words in the titles, clustering them by colors as described in the methodology. In Figure 3, these clusters signal possible research themes, i.e., sub-topics, within the main topic. The relative occurrence of each term is represented by the dot 

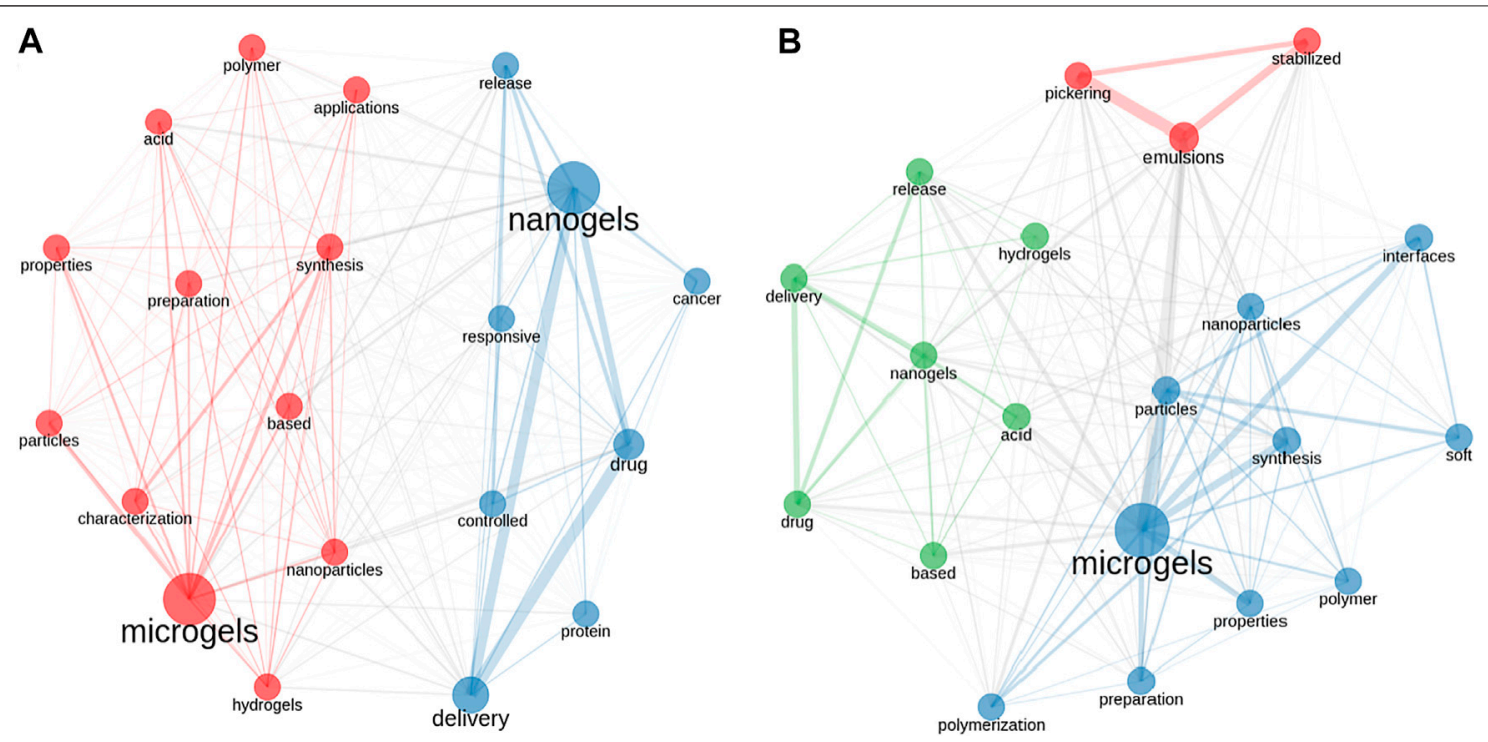

FIGURE 3 | Conceptual structure of the topic. Co-occurrence of words in titles. Bibliographic co-occurrence of the 20 most relevant words in titles between the years 2010-2020 for (A) the main topic "microgels and nanogels" and (B) the sub-topic "microgels and nanogels at interfaces and emulsions". Different colors correspond to different clusters or themes within the research topic. Different size correspond to total occurrences, and thickness of links correspond to the strength of the co-occurrence. Plots are produced with Biblioshiny.

size, while the label and the thickness of the links represent the strength of the co-occurrence in titles. Isolated terms with respect to the other 20 more relevant words, or terms with less than two links to other terms are removed. It is worth mentioning that these conceptual maps are generally more subjective than the descriptive results shown so far. The subjectivity is due to the different clustering algorithms with different criteria, and even within the same algorithm, different initial conditions as simple as the number of words considered (20 in our case), result in significantly different networks.

Therefore, the expert eye of a researcher familiar with the topic can detect possible artifacts, and this kind of analysis should be preferably used for comparison purposes between different research topics rather than for absolute analysis of a research topic. Moreover, a possible drawback of this clustering is that once a term is assigned to a cluster, it is not available for other clusters, while the co-occurrence with words in other clusters is still visible from the gray links in the plot. We compare the main topic "microgels and nanogels" and the sub-topic "microgels and nanogels at interfaces and emulsions" with the same analysis.

The first remarkable result, for both the main topic and subtopic, is the linking of the term microgels to rather non-specific terms, such as synthesis, properties, or applications. Moreover, the terms clustered around nanogel are biomedical related terms, such as drug or delivery, among others. In the main topic, Figure 3A, the biomedical-related terms clustering around nanogels are more specialized, such as protein and cancer. Therefore, it seems reasonable that authors tend to choose the term nanogel over microgel when trying to identify with this biomedical sub-topic, rather than classifying the microgels and nanogels from their molecular weight, as established in literature [11]. This analysis provides qualitative and quantitative ways of identifying conceptual aspects of the main topic, in this case, the choice of using the term microgel or nanogel. In the sub-topic "microgels and nanogels at interfaces and emulsions" (Figure 3B), we can see a bigger size of the bubble and label of the term microgel, compared to the term nanogel. This hints once more to an opportunity to improve the biomedical sub-topic in terms of better understanding the fundamental knowledge of microgels and nanogels at interfaces and emulsions, both being relevant aspects of the delivery and release of drugs in the human body. Furthermore, the term soft appears among the 20 most relevant, in the cluster related to the term microgel, pointing to a wide-spreading of these particles as model systems in the Soft Matter community. Finally, a third cluster is identified within the sub-topic, where the terms Pickering, emulsions and stabilized appear linked. This cluster arises as a separated theme weakly linked to the term interface, most likely pointing out a lack of fundamental and applied studies of microgels and nanogels at model (e.g., flat) interfaces. Therefore, this might present another research opportunity within the sub-topic.

One step more in extracting information of the conceptual structure are the thematic maps shown in Figure 4. This analysis first identifies the themes from co-occurrence of words in titles (as the clusters discussed above). Next, the themes are plotted as separated coloured circles in a 2D plot where the $x$-axis and $y$-axis represent the centrality and density of each theme, respectively. The theme appears more central to the research topic if there are more co-occurrences with words belonging to other themes. The theme becomes denser within the research topic when stronger co-occurrences happen within the theme. Thus, the more density of a theme, the more it is well-developed [5].

As a consequence, the density vs centrality produces a fourquadrant plot where the themes on the first quadrant (top-right) 


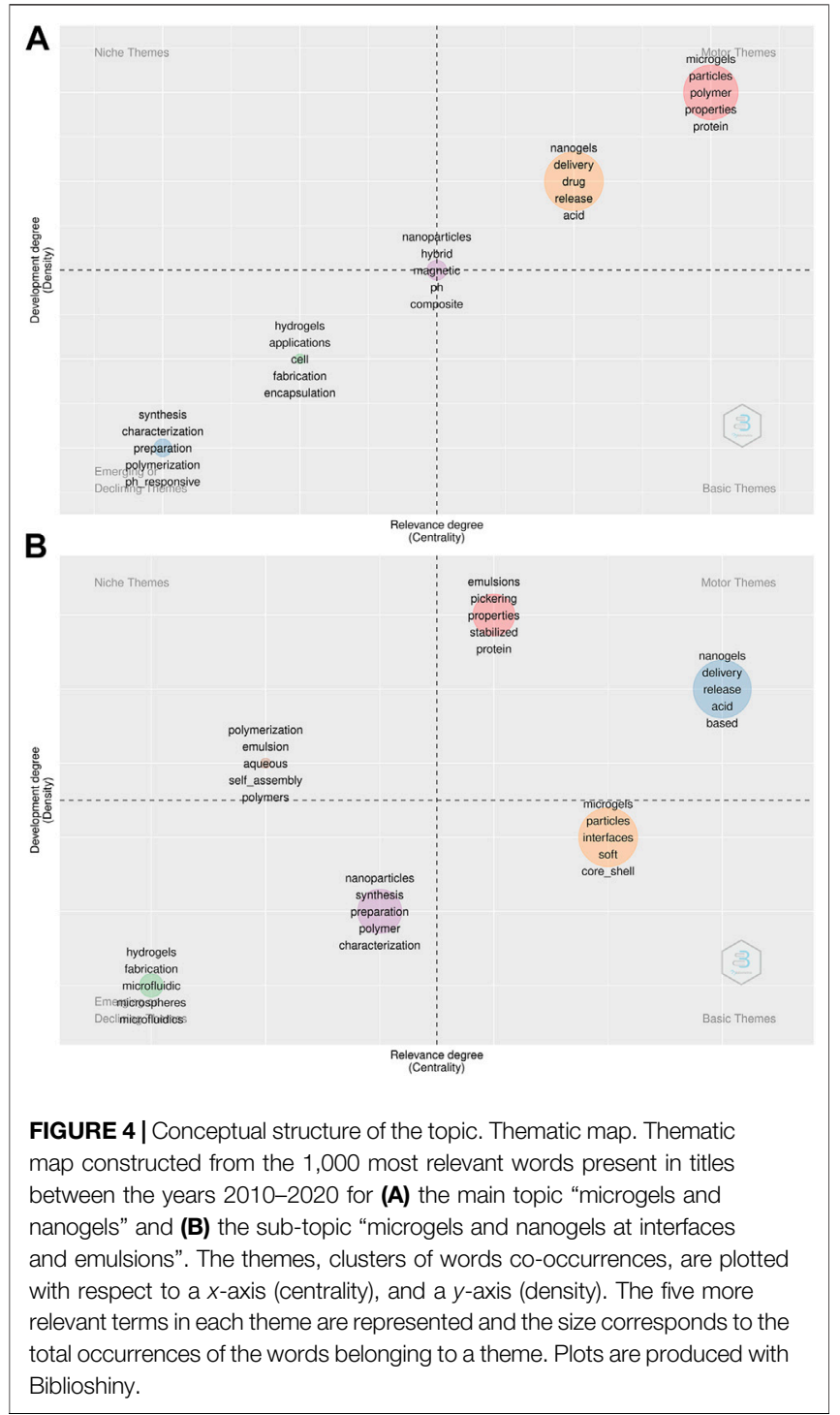

are more central and densely distributed, being considered the motor themes of the field of study. The second quadrant (top-left) is less central but dense, meaning that they are niche themes. The third quadrant (bottom-left) is less central and dense, being either emerging or disappearing themes. Finally, the fourth quadrant (bottom-right) represents central but less dense themes, the basic themes fundamental to the field of study [5]. This thematic map inherits the problems of reproducibility already mentioned for the co-occurrence of the words network, but since the themes are now separated entities, the links to other words in other themes are no longer visible. Nevertheless, useful information can be extracted once more with the combination of an expert eye and by comparing the main topic and the sub-topic between 2010 and 2020. When examining Figures $\mathbf{4 A , B}$, there is once more the separation between microgels as a general system, and nanogels specifically in drug delivery in both the main topic and sub-topic. The high centrality and relevance of these clusters is determined by the dot size of the theme, and thus, the occurrence of the words in the theme. In the main topic, Figure $4 \mathrm{~A}$, microgels and nanogels are motor themes of the research topic, with the microgels theme being more central than the nanogels theme. More specialized magnetic and composite nanoparticles appear as a medium central and dense theme. Applications regarding cells and encapsulation and publications focusing on synthesis appear either as emerging or declining themes. This trend might hint at a decline in research papers on pure synthesis, followed by an increase in the research on characterization and applications of microgels and nanogels. Regarding the sub-topic of "microgels and nanogels at interfaces and emulsions, in Figure 4B, the microgels theme appears less dense and central than the nanogels theme, even crossing to the quadrant of the basic themes. Moreover, in the sub-topic, the theme of Pickering emulsions, already identified in Figure 3B, appears as a less central but highly dense theme, showing again the apparent lack of sufficient connection between fundamental aspects of microgels and nanogels at interfaces, and their applications in Pickering emulsions. Therefore, this seems once more a possible and interesting research opportunity. Furthermore, the inversion of positions of the themes microgels and nanogels from Figure 4A to Figure 4B needs to be interpreted in combination with previous Figure 3. In Figure 3B, we see the dominant term microgels over nanogels, in size, and therefore frequency. However, in Figure 4B a new theme appears on Pickering emulsions, competing with the theme represented by the term microgel, and it is expected that when taken in combination, they would be denser and more developed than the theme on nanogels. This behavior hints at an easier interpretation of the thematic maps from an expert eye, and that they are useful in combination with the previous analysis. The theme on synthesis and pure characterization is a possible declining theme. At the same time, it is not possible to elucidate if the theme related to the term microfluidics also belongs to a declining or rather emerging theme. The latter seems plausible, as there is a slight increase of the term microfluidics over time when performing the word dynamics, as in Figures 2A-C, with keywords instead of words in titles (not shown in this manuscript). The small theme in the niche quadrant seems to be an artifact, as the term emulsion appears in singular and plural forms in different themes. Nevertheless, the thematic map was performed with Porter's word stemming algorithm [6], and all appearances of the term emulsion were changed to the plural form as discussed in the methodology in a previous step. Therefore, a better handling of the word stemming algorithm is needed. For example, while the thematic map allows choosing the Porter's word stemming algorithm [6], this is not an option for the cooccurrence networks in Figure 3. Regardless of these minor issues, the thematic map provides extra information about the conceptual structure of a research main topic and sub-topics. However, it needs a grain of salt when analyzing the results, as they are very dependent on the initial conditions of the analysis.

Finally, we analyze the collaboration networks through coauthorship occurrence in the bibliography items, as discussed in the methodology. We perform this analysis in two different ways, always restricting to the period 2010-2020: 1) by analyzing the collaboration between countries by number of publications (Table 1), and 2) by network maps of co-authors (Figure 5). 
TABLE 1 | Collaboration network of the main topic and sub-topic by countries. Table showing the collaboration for the main topic "microgels and nanogels" and the subtopic "microgels and nanogels at interfaces and emulsions". Constructed from the 10 more frequent collaborations based on publications between international research groups, restricted to the years 2010-2020. Countries are sorted in each row by alphabetic order.

Main Topic: Microgels and nanogels

\begin{tabular}{llc}
\hline Countries & & Publications \\
\hline China & United States & 259 \\
Germany & Netherlands & 84 \\
Canada & China & 68 \\
Germany & Russia & 67 \\
Germany & United States & 67 \\
South Korea & United States & 61 \\
China & United Kingdom & 60 \\
China & Germany & 59 \\
Egypt & Saudi Arabia & 55 \\
Germany & Spain & 51
\end{tabular}

\section{Sub-topic: Interfaces and emulsions}

\begin{tabular}{llc}
\hline Countries & & Publications \\
China & United States & 44 \\
Germany & Russia & 29 \\
China & United Kingdom & 21 \\
Canada & China & 19 \\
Egypt & Saudi Arabia & 19 \\
Germany & Netherlands & 18 \\
Germany & Switzerland & 15 \\
Germany & United States & 13 \\
China & Pakistan & 10 \\
France & United States & 10
\end{tabular}

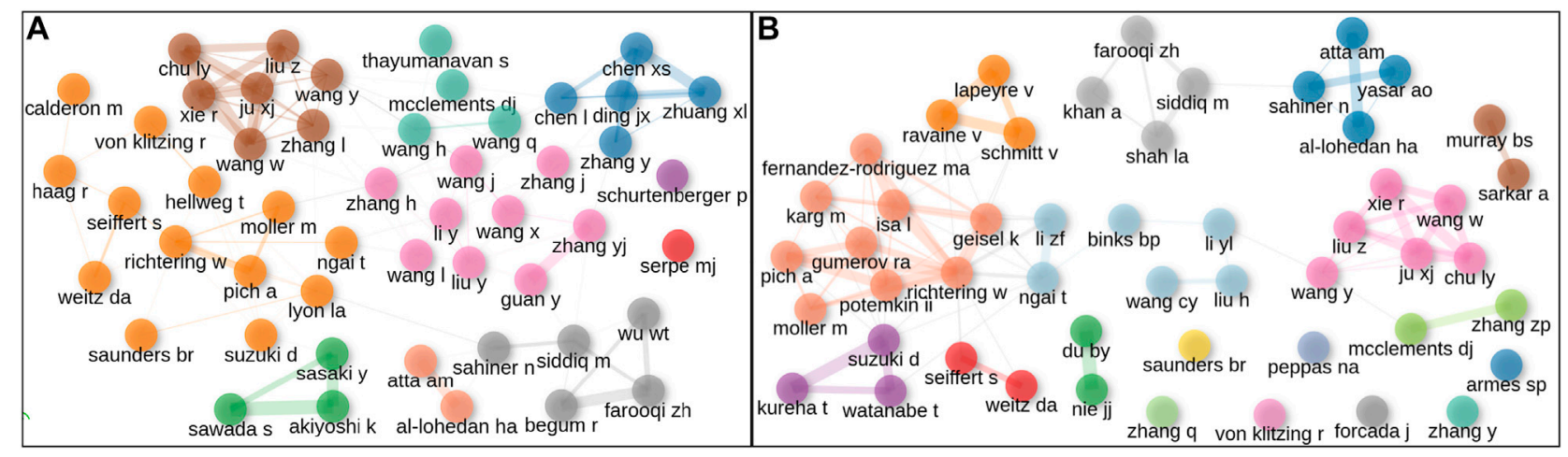

FIGURE 5| Social structure of the main topic and sub-topic. Collaboration network constructed from occurrence of co-authorship between the years 2010-2020, showing the 50 most frequent co-authorship for (A) the main topic "microgels and nanogels" and (B) the sub-topic "microgels and nanogels at interfaces and emulsions". Clusters of co-authors are identified with the same color and the thickness of the links correspond to the strength of the co-authorship. The isolated authors are an artifact of plotting 50 authors for the sake of clarity. Plots are produced with Biblioshiny.

The later share the same features as the maps in Figure 3, where the co-authors are identified as belonging to collaboration clusters with the same color, and thicker links between them mean frequent collaborations. It is worth mentioning that the networks were slightly modified to avoid overlapping names and to make it easier to see the links between co-authors. While restricting to the 50 more frequent authors in co-authorship already serves as a sort of ranking, this is inherent to constraining the total number of co-authors. In addition, we deactivated the sizing of the names with the total occurrence of authorship. We point once more that while the performance analysis of authors and journals is central to Biblioshiny, our goal is to provide tools to identify challenges and opportunities both theme- and collaboration-wise.

In Table 1, we restricted to the 10 most frequent collaborations between countries for clarity purposes. The countries are presented in alphabetic order. In this "bilateral" collaborations table, we observe that China-United States collaborations are by far the most frequent within the main topic, which is three times bigger than the next European collaboration, GermanyNetherlands. The difference in the sub-topic "at interfaces and emulsions" between China-United States is 1.5 times bigger than the next European collaboration Germany-Russia. Furthermore, China leads with 446 publications in the main topic, followed by the United States with 387, and Germany with 328. China and United States have both the biggest research communities due to their size and investment in research, but Germany stands here as appearing the most in this list, five times, signaling the significant specific weight of Germany in the main topic. Attending to the sub-topic at interfaces and emulsions, China still leads with 94 publications, followed closely by Germany with 75 , and the United States with 67. Thus, the specific weight of Germany in the sub-topic is higher than in the main topic. While the rest of the countries in the list are the so-called occidental, the appearance of the collaboration Egypt-Saudi Arabia is remarkable, which signals a clear team effort to study this kind of system by these countries. While this analysis is already helpful in understanding the collaborations between 
countries, these collaborations are usually restricted to a few research groups specialized in the discussed research topics. Therefore, a more detailed collaboration network featuring the names of co-authors, as shown in Figure 5, is helpful in combination with the previous table. As it was the case in Figure 3, in Figure $\mathbf{5}$ the algorithm of choice biases the normalization and the clustering process. In addition, the links between authors of different clusters are still visible in gray and remain biased by the normalization algorithm. Since analyzing the collaboration clusters represented by the names of the most frequent co-authors would introduce subjective analysis from our part, we only use this information to point out possible opportunities for potential collaborations, supported by the information displayed in Table 1 . The proposition of new collaboration opportunities relies on bringing together clusters that now appear separated by color and distance. Furthermore, these collaboration network maps serve two purposes, depending on whether the reader is a non-experienced or experienced researcher in this specific field. The non-experienced researcher can use it to identify frequent co-authors in the main topic or sub-topic, hopefully serving for a more efficient and smarter selection of publications, complementing in a faster way the perspectives of the authors in the multiple available reviews. Conversely, the experienced researcher can easily identify the names of their collaborators and have at a glance the names of other clusters of collaborators, which might open opportunities for new and fruitful collaborations. The methodology here is only a small sub-set of tools available in Biblioshiny, aimed at our goals of identifying opportunities theme- and collaboration-wise. We highly encourage the reader to import the datasets used in this work (see Supplementary Materials) to Biblioshiny and check the full table of collaborations. This exercise serves not only to identify the strengths already present, but also (as pointed during the reviewing process), "to aid in finding collaborators for bilateral calls for proposals, grants, and country-specific pre- and postdoctoral opportunities".

\section{CONCLUSION}

We used Biblioshiny to explore the bibliography on the main topic "microgels and nanogels" and compared to the sub-topic "microgels and nanogels at interfaces and emulsions". We constrained the analysis to the years 2010-2020 to identify current opportunities within the main topic and sub-topic, both theme- and collaboration-wise. We tracked the appearance of frequent words and themes that arise from the co-occurrence of words. The conceptual analysis of themes brings qualitative and quantitative ways of looking for conceptual connections within the topic. Thus, it provides a better overview of the themes around the research topic, helping to identify open opportunities, as for instance, how to improve current thematic connections. Thanks to this analysis, we concluded that the choice of using the terms nanogels or microgels, at least in the period 2010-2020, is motivated by wanting to identify with a biomedical sub-topic by using the term nanogels, while the term microgels is reserved for more general studies and applications. Moreover, the sub-topic of "microgels and nanogels at interfaces and emulsions" shows a smaller size of the nanogels cluster along with the terms drug delivery, compared to the rest of the sub-topic, pointing to an opportunity to bring more fundamental understanding of microgels and nanogels at interfaces and emulsions into biomedical applications. In addition, the weaker link between Pickering emulsions and microgels and interfaces points out a possible opportunity for the development of the field by bringing the physics of the microgels adsorbed at model interfaces into the Pickering emulsion applications. We also represented the collaboration map by countries, and a network map of coauthorship, enabling a descriptive view of the collaborations within the main topic and sub-topic. We think this kind of collaboration network mapping is useful for detecting solid and large collaboration clusters and highlighting the possibility of interacting with other clusters. Also, it will aid in finding potential collaborators in bilateral calls for proposals. Finally, the methodology and results presented here seem helpful for all stages of the research career. The bibliometric analysis was helpful to both acquire a comprehensive overview of the state-of-the-art of the main topic and sub-topics, and to find new opportunities, both regarding interesting or not sufficiently explored research themes, and present and potential collaborations.

\section{AUTHOR CONTRIBUTIONS}

All authors listed have made a substantial, direct, and intellectual contribution to the work and approved it for publication.

\section{FUNDING}

MAFR acknowledges funding from the projects PID2020116615RA-I00, MAT2017-82182-R, and grant IJC2018035946-I, funded by MCIN/ AEI /10.13039/501100011033, and FEDER "A way to make Europe" for the 2017 project. LA acknowledges funding from the Swiss National Science Foundation (SNCF) Grant PP00P2-172913/1. The manuscript publication fee is covered by the library services of ETH Zürich.

\section{ACKNOWLEDGMENTS}

Thanks to the University of Granada, and especially to Prof. Daniel Torres-Salinas, and the EC3metrics Spin-Off, for offering courses on bibliometrics applied to researchers.

\section{SUPPLEMENTARY MATERIAL}

The Supplementary Material for this article can be found online at: https://www.frontiersin.org/articles/10.3389/fphy.2021.754684/ full\#supplementary-material 


\section{REFERENCES}

1. Aria M, and Cuccurullo C. Bibliometrix : An R-Tool for Comprehensive Science Mapping Analysis. J Informetrics (2017) 11:959-75. doi:10.1016/ j.joi.2017.08.007

2. Baker WO. Microgel, A New Macromolecule. Ind Eng Chem (1949) 41:511-20. doi:10.1021/ie50471a016

3. Blondel VD, Guillaume J-L, Lambiotte R, and Lefebvre E. Fast Unfolding of Communities in Large Networks. J Stat Mech (2008) 2008:P10008. doi:10.1088/1742-5468/2008/10/p10008

4. Fruchterman TMJ, and Reingold EM. Graph Drawing by Force-Directed Placement. Softw Pract Exper (1991) 21(11):1129-64. doi:10.1002/ spe.4380211102

5. Cobo MJ, López-Herrera AG, Herrera-Viedma E, and Herrera F. An Approach for Detecting, Quantifying, and Visualizing the Evolution of a Research Field: A Practical Application to the Fuzzy Sets Theory Field. J Informetrics (2011) 5: 146-66. doi:10.1016/j.joi.2010.10.002

6. Porter MF. An Algorithm for Suffix Stripping. Program (1980) 14:130-7. doi:10.1108/eb046814

7. Sterling T, Anderson M, and Brodowicz M. "MapReduce". In: High Performance Computing. Ed. by S Thomas, A Matthew, and B Maciej. Boston: Morgan Kauf mann, 2018, pp. 579-89. doi:10.1016/B978-0-12420158-3.00019-8

8. Pelton R. Temperature-sensitive Aqueous Microgels. Adv Colloid Interf Sci (2000) 85:1-33. doi:10.1016/S0001-8686(99)00023-8
9. Zhilin DM, and Pich A. Nano- and Microgels: a Review for Educators. Chem Teach Int (2021) 3:155-67. doi:10.1515/cti-2020-0008

10. Pelton RH, and Chibante P. Preparation of Aqueous Latices with N-Isopropylacrylamide. Colloids Surf (1986) 20:247-56. doi:10.1016/01666622(86)80274-8

11. Graham NB, and Cameron A. Nanogels and Microgels: The New Polymeric Materials Playground. Pure Appl Chem (1998) 70:1271-5. doi:10.1351/ pac199870061271

Conflict of Interest: The authors declare that the research was conducted in the absence of any commercial or financial relationships that could be construed as a potential conflict of interest.

Publisher's Note: All claims expressed in this article are solely those of the authors and do not necessarily represent those of their affiliated organizations, or those of the publisher, the editors and the reviewers. Any product that may be evaluated in this article, or claim that may be made by its manufacturer, is not guaranteed or endorsed by the publisher.

Copyright $(\odot 2021$ Fernandez-Rodriguez and Alvarez. This is an open-access article distributed under the terms of the Creative Commons Attribution License (CC BY). The use, distribution or reproduction in other forums is permitted, provided the original author(s) and the copyright owner(s) are credited and that the original publication in this journal is cited, in accordance with accepted academic practice. No use, distribution or reproduction is permitted which does not comply with these terms. 